\title{
ESTUDO ELETROMANOMÉTRICO DO ESÔFAGO EM PORTADORES DA DOENÇA DE CHAGAS EM SUA FORMA INDETERMINADA
}

\section{MANOMETRIC FINDINGS OF THE ESOPHAGUS OF PATIENTS WITH INDETERMINATE FORM OF CHAGAS'S DISEASE}

\author{
Eduardo Crema, TCBC - MG ${ }^{1}$ \\ Renata Mônica de Oliveira ${ }^{2}$ \\ Ana Marcia Werneck ${ }^{2}$ \\ Luiz Augusto Figueiredo Cruvinel ${ }^{2}$ \\ Juverson Alves Terra Júnior ${ }^{3}$ \\ Alex Augusto Silva ${ }^{4}$
}

\begin{abstract}
RESUMO: Objetivos: Avaliar as alterações manométricas dos esfíncteres superior (ESE) e inferior do esôfago, bem como a motilidade de seu corpo, em pacientes com a forma indeterminada da Doença de Chagas. Método: Foram considerados 37 pacientes portadores da Doença de Chagas, assintomáticos, que apresentavam eletrocardiograma, enema opaco e radiografia contrastada do esôfago sem alterações características da doença (forma indeterminada). Estes foram submetidos à eletromanometria do esôfago em que foram analisados dados sobre a pressão dos esfíncteres e ondas peristálticas do corpo do esôfago. Resultados: Detectouse uma diminuição da média da amplitude de contração do corpo do esôfago $(\mathrm{p}=0,03)$ nos portadores de ondas sincrônicas quando comparados com os portadores de ondas assincrônicas. A comparação da média das pressões máximas do ESE nos pacientes portadores de ondas sincrônicas foi significativamente maior $(\mathrm{p}=0,02)$ que a média encontrada nos portadores de ondas assincrônicas. Conclusão: Encontrou-se um elevado número $(48,65 \%)$ de portadores de ondas sincrônicas em pacientes com a forma indeterminada da Doença de Chagas; notou-se uma redução da média da amplitude da contração do corpo do esôfago em portadores de ondas sincrônicas e observou-se que a média das pressões máximas do ESE é maior nos pacientes com ondas sincrônicas $(207,14 \mathrm{mmHg})$ quando comparada com os portadores de ondas assincrônicas $(142,44 \mathrm{mmHg})$. Dessa forma propomos uma discussão sobre a classificação atual da Doença de Chagas.
\end{abstract}

Descritores: Doença de Chagas; Manometria; Esôfago; Junção esofagogástrica; Diagnóstico.

\section{INTRODUÇÃO}

A doença de Chagas é uma doença multissistêmica causada pelo Trypanosoma cruzi e que acomete cerca de 5 a 8 milhões de pessoas no Brasil $^{1}$. De seus portadores, aproximadamente $10 \%$ desenvolvem a forma esofágica da doença ${ }^{2}$, que se manifesta de várias formas na dependência do grau da esofagopatia. Um dos principais achados anatomopatológicos do megaesôfago chagásico é a desnervação dos plexos mioentéricos ao longo de todo esôfago médio e distal, ou seja, corpo e esfíncter inferior esofágico ${ }^{1}$. Köeberle ${ }^{3}$ demonstrou que $90 \%$ dos neurônios do plexo de Auerback estão destruídos em pacientes com megaesôfago e que em pacientes Chagásicos, com esôfago não dilatado, já é observa-

\footnotetext{
1. Professor Titular em Cirurgia do Aparelho Digestivo da Faculdade de Medicina do Triângulo Mineiro (CAD/FMTM).

2. Doutorandos da Faculdade de Medicina do Triângulo Mineiro.

3. Residente da CAD/FMTM.

4. Professor Adjunto Responsável pela CAD/FMTM.
}

\section{Recebido em 13/06/2003}

Aceito para publicação em 16/12/2003

Trabalho realizado na Faculdade de Medicina do Triângulo Mineiro - FMTM. 
da alguma desnervação. Segundo este autor, alterações motoras surgem quando $50 \%$ destas estruturas neurais já estão comprometidas. Conceitualmente os portadores da doença de Chagas na sua forma indeterminada devem apresentar: 1) positividade de exames sorológicos e/ou parasitológicos; 2) ausência de sintomas e/ou sinais da doença; 3 ) eletrocardiograma convencional normal e, 4) coração, esôfago e cólons radiologicamente normais ${ }^{4}$.

A disfagia lentamente progressiva é o sintoma mais característico da esofagopatia chagásica. Sintomas, também comuns no inicio do quadro, como regurgitação e dor retroesternal são conseqüentes à alteração motora provocada pela desnervação parcial do órgão ${ }^{3}$. Na investigação desses sintomas, exames radiográficos, endoscópicos, citilográficos e manométricos são utilizados de rotina. A eletromanometria do esôfago torna-se essencial no estudo da doença de Chagas, pois é capaz de detectar alterações esofágicas precoces em indivíduos assintomáticos ${ }^{5,6}$, por permitir um estudo detalhado da função motora do esôfago, bem como uma análise da localização, extensão e pressão de seus esfíncteres.

Nos portadores de megaesôfago chagásico desaparecem as ondas assincrônicas indicativas de um movimento peristáltico harmônico e propulsivo. Nestes pacientes, após a deglutição surgem ondas sincrônicas de pressão, conhecidas por alguns autores como aperistalse $\mathrm{e}^{7-10}$ por todo o esôfago, o que dificulta a condução do bolo alimentar ao estômago. Além disso, não é observada a queda da pressão no esfíncter inferior do esôfago após a deglutição, caracterizando a abertura incompleta ou não abertura do esfíncter (acalasia) ${ }^{7}$.

Oliveira et $a l^{7}$, analisando pacientes chagásicos e sem dilatação do esôfago, observaram que 53,5\% apresentavam disfagia e que em apenas $27,9 \%$ essa disfagia era persistente. Os achados eletromanométricos de seu estudo foram: $37,2 \%$ dos pacientes apresentaram motilidade normal; $7 \%$ apresentavam ondas peristálticas polifásicas; $21 \%$ tinham aperistalse do esôfago com diminuição do relaxamento do esfíncter inferior do esôfago (EIE) e 34,8\% apresentavam aperistalse e acalasia do EIE. Pinotti ${ }^{11}$ refere uma diminuição da amplitude da onda de contração do esôfago em Chagásicos, quando analisados o terço superior, médio e inferior do esôfago.

Os portadores da forma digestiva da doença de Chagas por apresentarem elevada parasitemia, têm sido tratados rotineiramente em nosso serviço com benzonidazol na tentativa de evitar a progressão da doença. Propõe-se, neste estudo, identificar, entre os pacientes com a forma indeterminada da doença, aqueles que apresentam alterações eletromanométricas características da esofagopatia chagásica que poderão se beneficiar com o tratamento medicamentoso específico, uma vez que o tratamento cirúrgico é apenas paliativo.

O objetivo deste estudo é avaliar as alterações eletromanométricas dos esfíncteres superior (ESE) e inferior (EIE) do esôfago, bem como a motilidade de seu corpo em pacientes portadores da doença de Chagas em sua forma indeterminada.

\section{MÉTODO}

Foram considerados 37 pacientes portadores da doença de Chagas comprovados por pelo menos dois testes sorológicos positivos (Imunofluorecência indireta, Machado Guerreiro, Elisa ou Hemoaglutinação), que além de assintomáticos apresentavam eletrocardiograma, enema opaco e radiografia contrastada do esôfago sem as alterações características da doença (forma indeterminada).

Os pacientes foram submetidos à eletromanometria do esôfago através de um cateter plástico introduzido pelo orifício nasal até o estômago. O cateter é composto por seis canais (três orifícios na extremidade mais distal, e os outros três com espaçamento de $5 \mathrm{~cm}$ entre eles) por onde perfundiuse água destilada a uma velocidade constante de $0,6 \mathrm{ml} / \mathrm{min} /$ canal. Após a comprovação de que todos os canais encontravam-se situados ao nível do abdome, o cateter foi retirado intermitentemente, centímetro a centímetro. Dessa forma, ao final do procedimento, foi possível analisar simultaneamente as contrações peiristálticas dos terços superior, médio e inferior do esôfago, além de registrar a abertura da zona de alta pressão (ZAP), localizada na transição esôfago-gástrica e avaliar a pressão dos esfíncteres superior e inferior do esôfago. Qualquer variação da pressão intraluminar era traduzida como obstáculo ao fluxo de água. O registro das alterações pressóricas foi feito através de um polígrafo e decodificado por um "Software" próprio (Polygram for Windows versão 2.02).

Dessa forma foi possível analisar os dados quanto à localização, extensão e pressão dos esfíncteres, assim como a amplitude das ondas peristálticas do corpo do esôfago. Os dados obtidos foram avaliados pelo programa estatístico teste "t-student". 
Tabela 1 - Medidas descritivas referentes ao esfincter inferior do esôfago, quanto a localização (EIE-LOC), extensão (EIE-EXT) e pressão média (EIE-PMR) comparando os portadores de contrações sincrônicas $(S)$ e assincrônicas (A).

\begin{tabular}{|l|l|l|l|l|l|}
\cline { 2 - 6 } \multicolumn{1}{c|}{} & \multicolumn{2}{l|}{ MÉDIA } & \multicolumn{2}{l|}{ DESVIO PADRÃO } \\
\hline VARIÁVEL & S & A & A & S & VALOR-P \\
\hline EIE-LOC & 44,00 & 44,29 & 2,99 & 3,56 & 0,82 \\
EIE-EXT & 2,89 & 2,29 & 0,91 & 0,61 & 1,00 \\
EIE-PMR & 19,63 & 17,24 & 6,02 & 5,60 & 0,26 \\
\hline
\end{tabular}

\section{RESULTADOS}

Após analise manométrica do esôfago os pacientes foram reunidos em dois grupos: grupo 1, constituído por 19 pacientes que apresentavam ondas assincrônicas $(51,35 \%)$ ao teste manométrico; e o grupo 2, constituído de 18 pacientes em que foram detectadas ondas sincrônicas $(48,65 \%)$.

Observou-se entre os pacientes estudados um número considerável de portadores de ondas bifásicas ou polifásicas $(56,7 \%)$ ao exame eletromanométrico.

Quando comparados os grupos quanto ao sexo e idade não foram detectadas diferenças significativas, sendo, portanto os grupos homogêneos. O mesmo pode-se dizer quando avaliados os dados quanto à localização e extensão dos esfíncteres, bem como quando analisamos a pressão do EIE (Tabela 1).

Detectou-se uma diminuição estatisticamente significante da média da amplitude das con-

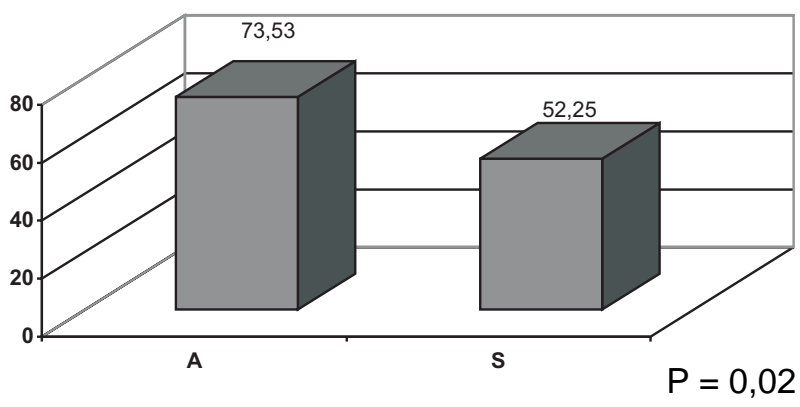

Figura 1 - O gráfico um representa a comparação das médias da amplitude de contração do corpo do esôfago entre os pacientes com ondas assincrônicos $(A)$ e sincrônicos $(S)$.

trações peristálticas do corpo do esôfago em pacientes portadores de ondas sincrônicas, quando comparadas aos portadores de ondas assincrônicas (Figura 1).

A comparação da média das pressões máximas do esfíncter superior do esôfago nos pacientes portadores de ondas sincrônicas ao teste manométrico foi significativamente maior $(204,17 \mathrm{mmHg})$ que a média encontrada nos portadores de ondas assincrônicas $(142,44 \mathrm{mmHg})$ (Figura 2).

\section{DISCUSSÃO}

Sabe-se que a desnervação que ocorre na doença de Chagas é a responsável pela redução da amplitude de contração e pela alteração da peristalse harmônica responsável pela condução do bolo alimentar ao estômago. Muitos estudos têm procurado demonstrar essas alterações funcionais da esofagopatia

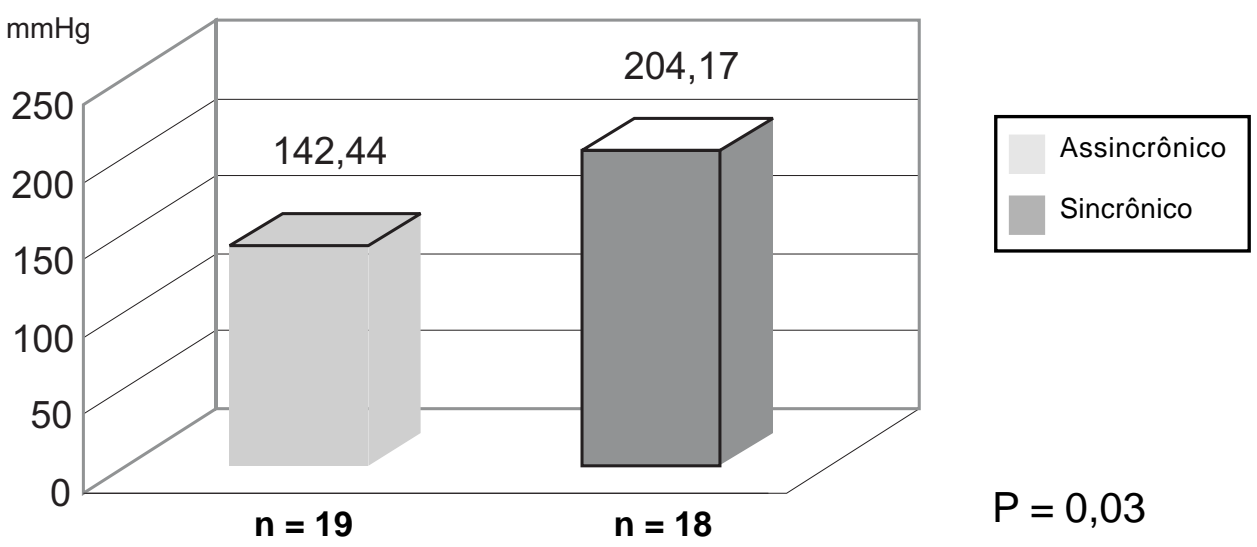

Figura 2 - O gráfico dois mostra a comparação das médias das pressões máximas do esfíncter superior entre pacientes com ondas sincrônicas e assincrônicas ao teste manométrico. 
Chagásica através da análise eletromanométrica do esôfago ${ }^{5-8,10-13}$.

Oliveira $e t a l^{7}$, analisando pacientes chagásicos com e sem dilatação do esôfago, observou alterações na eletromanometria como a presença de ondas polifásicas $(7 \%)$ e aperistalse $(55,8 \%)$ acompanhadas ou não de relaxamento do esfíncter inferior do esôfago. Embora este estudo tenha encontrado resultados semelhantes no que se refere a peristalse, observou-se entre os pacientes estudados um número consideravelmente maior de portadores de ondas bifásicas ou polifásicas à eletromanometria $(56,7 \%)$.

Obteve-se neste estudo uma redução significante da média das amplitudes de contração do corpo esofágico $(52,25 \mathrm{mmHg})$ entre os pacientes portadores de ondas sincrônicas quando comparados com os portadores de ondas assincrônicas, resultados semelhantes aos referidos por Dantas ${ }^{5,8}$.

Lemme $e a l^{6}$, comparando a pressão EIE entre pacientes chagásicos, portadores de acalasia idiopática e grupo controle, notaram que os pacientes com acalasia idiopática apresentavam maior pressão no EIE quando comparados aos pacientes chagásicos, e que estes não diferiam do grupo controle ${ }^{6}$. Dantas ${ }^{9}$ estudando pacientes chagásicos observou que embora a pressão no EIE tenha sido pouco maior nos pacientes com peristalse (21 mmHg) quando comparados aos pacientes com aperistase $(19 \mathrm{mmHg})$, essa diferença não foi estatisticamente significante. Neste estudo, da mesma forma, a pressão do EIE em portadores de ondas sincrônicas $(19,63 \mathrm{mmHg})$ não diferiu, de forma significativa, dos portadores de ondas assincrônicas $(17,24 \mathrm{mmHg})$.
Dantas $^{9}$, ao analisar a pressão do ESE, notou que pacientes chagásicos e sem dilatação apresentavam maior pressão do esfíncter superior do esôfago, quando comparados a voluntários normais e pacientes com acalasia idiopática. Da mesma forma, neste estudo observou-se que o grupo de pacientes com ondas sincrônicas a manometria apresentava a média das pressões máximas do ESE estatisticamente maior que o grupo de pacientes com ondas assincrônicas.

Para participar da forma indeterminada da doença de Chagas, os portadores da doença não deveriam apresentar comprometimento morfológico ou funcional de seus órgãos, em especial coração, esôfago e cólon. Por este estudo ter detectado grande número de alterações nos portadores da forma indeterminada, discute-se então se $48,65 \%$ dos pacientes estudados que apresentaram ondas sincrônicas devem ser excluídos dessa classificação, pois estes apresentaram alterações funcionais traduzidas pela presença de ondas sincrônicas e pela redução na amplitude de contração do corpo esofágico.

Com este estudo podemos concluir que:

- existe um elevado número $(48,65 \%)$ de portadores de ondas sincrônicas entre os pacientes com a forma indeterminada da doença de Chagas.

- notou-se uma redução da média de amplitude de contração do corpo esofágico nos pacientes portadores de ondas sincrônicas quando comparados aos portadores de ondas assincrônicas e,

- observou-se que a média das pressões máximas do ESE é maior nos portadores de ondas sincrônicas que nos portadores de ondas assincrônicas.

\begin{abstract}
Objetive: To study the manometric findings of the lower esophageal sphincter(LES) and upper esophageal sphincter (UES) as well as the motility of the body of esophagus of patients with indeterminate form of Chagas'disease. Methods: The patients had positive serological for Chagas' disease but were asymptomatic and the eletrocardiogram, colon and esophagus showed no radiological findings typical of Chagas' disease. Thirty-seven subjects were submitted to the manometric study of the esophagus to evaluate the pressure of the sphincteres and peristalsis of the body of the esophagus. Results: There was a reduction of the average of the amplitude of the esophageal contraction waves $(p=0.03)$ at the body of the esophagus of subjects with synchronic waves when compared with subjects with asynchronic waves. The average of the maximum pressure value of the UES in synchronic group was significantly higher $(p=0.02)$ than the avarege found in subjects with asynchronic waves. Conclusion: It was found a great number of subjects with synchronic waves in the undeterminated form of Chagas'disease (48.65\%). The average of the amplitude of esophageal waves was smaller in subjects with synchronic waves. The avarage of maximum UES pressure is higher in subjects with synchronic waves than in subjects with asynchronic waves.
\end{abstract}

Key Words: Chagas'disease; Manometry; Esophagus; Esophagogastric junction; Diagnosis 


\section{REFERÊNCIAS}

1. Ferreira MS, Lopes ER, Chapadeiro E, et al - "Doença de Chagas". In Veronesi R, Focaccia R (eds) - Tratado de Infectologia. $4^{a}$ Edição, São Paulo, Atheneu, 1999, v. 2, pp.1175-1211.

2. Coura JR, Anunziato N, Willcox HP - Morbidade da Doença de Chagas. I - Estudo de casos procedentes de vários estados do Brasil, observados no Rio de Janeiro. Mem Inst Oswaldo Cruz,1983,78(3):363-372.

3. Koberle F - Chagas' disease and Chagas' syndromes: the pathology of American trypanosomiasis. Adv Parasitol, 1968, 6:63-116.

4. I Reunião de Pesquisa Aplicada em Doença de Chagas - Validade do conceito da forma indeterminada da doença de Chagas - Rev Bras Med Trop, 1985, 18(1):46.

5. Dantas RO, Deghaide NH, Donadi EA - Esophageal manometric and radiologic findings in asymptomatic subjects with Chagas' disease. J Clin Gastroenterol, 1999, 28(3):245-248.

6. Lemme EM, Domingues GR, Pereira VL, et al. - Lower esophageal sphincter pressure in idiophathic achalasia and Chagas disease-related achalasia. Dis Esophagus, 2001, 14(3-4):232-234.

7. Oliveira RB , Rezende JF, Dantas RO, et al. - The spectrum of esophageal motor disorders in Chagas' disease. Am J Gastroenterol, 1995, 90(7):1119-1124.
8. Dantas RO, Godoy RA, Padovan W, et al. - A contratilidade do esôfago na esofagopatia chagasíca. GED gastroenterol endosc dig, 1983, 2(1):26-28.

9. Dantas RO - Relação entre a motilidade e sintomas esofágicos com a pressão do esfíncter inferior do esôfago na doença de Chagas. GED gastroenterol endosc dig, 1993, 12(1):23-26.

10. Dantas RO - Upper esophageal sphincter pressure in patients with Chagas' disease and primary achalasia. Braz J Med Biol Res, 2000, 33 (5): 545-551.

11. Pinotti HW, Felix VN, Zilberstein B, et al. - Surgery complications of Chagas' disease: megaesophagus, achalasia of the pylorus and cholelithiasis. World $\mathbf{J}$ Surg, 1991,15(2):198-204.

12. Dantas RO - Vigorous achalasia in Chagas' disease. Dis Esophagus, 2002, 15(4):305-308.

13. Lovecek M, Duda M, Gryga A, et al. - Manometry in esophageal achalasia. Dis Rozhl Chir, 2002,81(4):183187.

Endereço para correspondência:

Eduardo Crema

Rua Duque de Caxias, 165- Apto 301

38022-180 - Uberaba- MG

eduardocremafmtm@mednel.com.br 